Mots. Les langages du politique

\title{
Du candidat au président. Panorama logométrique de François Hollande
}

From the candidate to the president. Logometric panorama of François

Hollande's speech

De candidato a presidente. Panorama logométrico de François Hollande

Damon Mayaffre

\section{(2) OpenEdition}

\section{Journals}

Édition électronique

URL : http://journals.openedition.org/mots/22479

DOI : $10.4000 /$ mots. 22479

ISSN : 1960-6001

Éditeur

ENS Éditions

Édition imprimée

Date de publication : 15 octobre 2016

Pagination : 81-92

ISBN : 978-2-84788-850-8

ISSN : 0243-6450

Référence électronique

Damon Mayaffre, "Du candidat au président. Panorama logométrique de François Hollande », Mots Les langages du politique [En ligne], 112 | 2016, mis en ligne le 15 octobre 2018, consulté le 02 janvier 2020. URL : http://journals.openedition.org/mots/22479; DOI : 10.4000/mots.22479 


\section{Du candidat au président. Panorama logométrique de François Hollande}

Pour des raisons institutionnelles et historiques, le pouvoir des présidents quinto-républicains est devenu un pouvoir logocratique et performatif; ce qui est un pouvoir majeur mais difficile à manier.

En effet, sous la Cinquième République, les présidents français ne sont pas les chefs d'une majorité parlementaire, et ne sont donc pas toujours dans la capacité de gouverner le pays par la loi (i.e. la contrainte législative); les situations de cohabitation étant le cas de figure le plus extrême de cette réalité constitutionnelle qui peut faire du président un souverain omnipotent, aux pouvoirs constitutionnels élargis mais sans majorité pratique et effective, un roi-élu puissant ${ }^{1}$ mais sans gouvernement.

Par ailleurs, à partir des années quatre-vingt, et peut-être depuis la disparition de l'Empire colonial, la politique de la France, en tant qu'État-Nation, ne peut se comprendre sans les contraintes et dépendances fortes au sein du marché mondial (OMC, G 20), d'organismes internationaux toujours mieux structurés (ONU, OTAN, TPI, etc.) et évidemment de l'Union européenne, de sa Banque centrale indépendante ou de ses directives; il y a là une réalité politique et historique que cette contribution ne saurait aborder mais qui de facto amoindrit les marges de manœuvre réelles du président français tout en augmentant son pouvoir symbolique.

Le vrai pouvoir des présidents, dans le quotidien de leur charge, est donc ailleurs que dans la prise de décision². Il est dans sa capacité à parler pour agir, à représenter les Français par la parole et figurer le pays par le verbe, à performer une politique par des «je veux» ou des «il faudrait», à entrainer le peuple par des professions de foi ou des déclarations solennelles. Pour reprendre la théorie bourdieusienne, le président est celui qui détient le privilège de saisir le

1. Outre le pouvoir du discours que l'on plaidera ici, on connait les ressorts de la puissance des présidents gaulliens, élus au suffrage universel, particulièrement dans des conditions exceptionnelles : chef des armées en temps de guerre (art. 15), plein pouvoir en temps de crise (art. 16), pouvoir discrétionnaire de dissolution en cas de différends parlementaires (art. 12). Voir par exemple Carcassonne (2011).

2. Nous écartons donc les situations de guerre et le régime d'exception.

Université de Nice-Sophia Antipolis, Bases, Corpus, Langage (CNRS, UMR 7320) mayaffre@unice.fr

Mots. Les langages du politique $\mathrm{n}^{\circ} 112$ novembre $2016 \bullet 81$ 
skeptron - l'élection au suffrage universel lui confère ce pouvoir - pour prendre la parole au nom de tous en espérant être cru ou obéi, en espérant convaincre ou dissuader, en transformant le monde par les mots (Bourdieu, 1982).

Sur cette scène logocratique bien huilée, qui va de la déclaration de candidature de l’impétrant aux vœux télévisés du président élu, et du débat de l'entre-deux-tours aux conférences de presse de l'Élysée, il existe un hiatus souvent pointé et autour duquel tourne l'inquiétude démocratique : la (dis) continuité de la parole politique entre le candidat et l'élu, entre les discours de campagne et les discours du trône, entre les promesses électorales et les grand-messes élyséennes; hiatus qui touche à la confusion lorsque le candidat déclaré est président-sortant ou que le président en exercice est d'évidence candidat à sa réélection.

Cette contribution tentera ainsi de comparer les discours des candidats à la présidentielle et des élus à l'Élysée en s'arrêtant principalement sur François Hollande. Cette comparaison est méthodologiquement incertaine puisque l'analyse du discours depuis ses origines a insisté sur la prégnance des conditions d'énonciation dans les prises de parole : il serait ainsi techniquement impossible, au fond, pour un président qui s'exprime sous les ors de l'Élysée, de produire le même discours qu'un candidat battant la campagne dans des meetings électoraux. Et cette conviction forte de l'analyse du discours est renforcée par les études génériques en linguistique textuelle qui posent avec Bakhtine, Maingueneau, Adam ou Rastier que le genre d'un discours électoral ne saurait ressembler - sans que l'on ne puisse rien conclure sur le locuteur à un discours institutionnel.

C'est donc avec prudence que nous avons rassemblé les discours du candidat Hollande en 2012 (tous ses discours de meeting et des interviews dans la presse) et les discours du président Hollande lors des quatre premières années de son mandat (ses discours grand public, ses allocutions télévisées et interviews dans la presse) ; pour une mise en perspective, nous convoquerons aussi tous les discours de campagne de Nicolas Sarkozy en 2007 et en 2012 ; et un demi-millier de discours présidentiels prononcés par François Hollande et Nicolas Sarkozy donc, mais aussi Charles de Gaulle, Georges Pompidou, Valéry Giscard d’Estaing, François Mitterrand et Jacques Chirac à l'Élysée3.

Quel fut le discours du candidat Hollande en 2012 ? Quel est le discours du président Hollande depuis qu’il est chargé des destinées du pays? Sur la base de cette problématique, nous mobilisons les outils de la logométrie et les logi-

3. Le corpus rassemble ainsi 722 discours équivalents à 2643567 occurrences. Les principales sources sont le site de l’Élysée 〈http://www.elysee.fr/〉 (consulté le 20 juin 2016), le site gouvernemental Vie publique 〈http://www.vie-publique.fr/〉 (consulté le 20 juin 2016) et les sites de campagne des candidats : 〈http://francoishollande.fr〉 (renvoi immédiat sur la page Facebook de FH, 30 août 2016), 〈http://www.lafranceforte.fr〉 (site désactivé, 30 août 2016). Beaucoup de ces discours enrichissent l'Observatoire du discours présidentiel que nous avons mis en ligne: 〈http://mesure-du-discours.unice.fr/〉(consulté le 20 juin 2016). 
ciels Hyperbase 10.0-2016 et Hyperbase Web Edition «http:// hyperbase.unice. $\mathrm{fr} />$ (consulté le 20 juin 2016) afin, non pas d'administrer la preuve statistique à l'hypothèse politico-linguistique, mais d'objectiver des parcours interprétatifs de lecture dans ce gros corpus. Ce sont principalement les thématiques du discours qui seront passées au crible grâce à des protocoles méthodologiques décrits pour partie dans le numéro 108 de Mots (Ben Hamed et Mayaffre, 2015) : corrélats sémantiques, réseaux cooccurrentiels et distance intertextuelle.

\section{Cartographie d'une campagne : Hollande versus Sarkozy en 2012}

L'agenda. Le sort d'une élection se joue non seulement sur les prises de position des candidats mais plus encore peut-être, en amont, sur l'agenda setting (à l'origine, McCombs, Shaw, 1972) qu'ils sont capables d'imposer au corps électoral; en ce sens, la presse joue un rôle fondamental en mettant sur le devant de la scène médiatique les thématiques à débattre, comme l'a montré par exemple le déroulement de la campagne présidentielle 2002 : à grand renfort des principaux organes de presse, les candidats Chirac et Le Pen ont réussi à éliminer dès le premier tour le candidat Jospin en labourant la thématique sécuritaire sur laquelle le candidat socialiste était moins à l'aise que sur le terrain économique et social (Née, 2012).

Aussi, nous nous proposons d'abord de définir à grands traits l'agenda de la campagne 2012 en analysant tous les meetings électoraux ainsi que plusieurs interviews de François Hollande et de Nicolas Sarkozy entre janvier et mai 2012; ce choix restreint à deux candidats limite évidemment le point de vue, mais la qualification de l'un et de l'autre pour le deuxième tour permet de le justifier.

Sur le graphe4, trois thématiques majeures se distinguent en 2012 sans qu'aucune ne semble l'emporter dans le discours; cette campagne nous apparait comme assez généraliste, sinon molle, à la manière de 1988 peut-être, et non pas mono-polarisée comme celle de 2002 sur la sécurité intérieure ou multi-polarisée comme celle de 2007 (Barbet et Mayaffre, 2009; Labbé, Monière, 2013).

À droite du graphique se distingue la thématique économique et sociale autour par exemple des mots production, industrie, dépense, compétitivité, travailleur, retraite, fiscalité, etc.

En haut à gauche, une constellation lexicale organise des réflexions poin-

4. Les principes du graphe ont été exposés, en trois temps, notamment par Guaresi (2015) et précédemment par Viprey (2006) : 1) sélection des 300 substantifs les plus utilisés dans le corpus ; 2 ) construction d'une matrice de cooccurrences pour mesurer la relation que ces substantifs entretiennent entre eux; 3 ) projection vectorielle des profils concurrentiels des mots grâce à l'analyse factorielle des correspondances (Benzécri, 1973). 


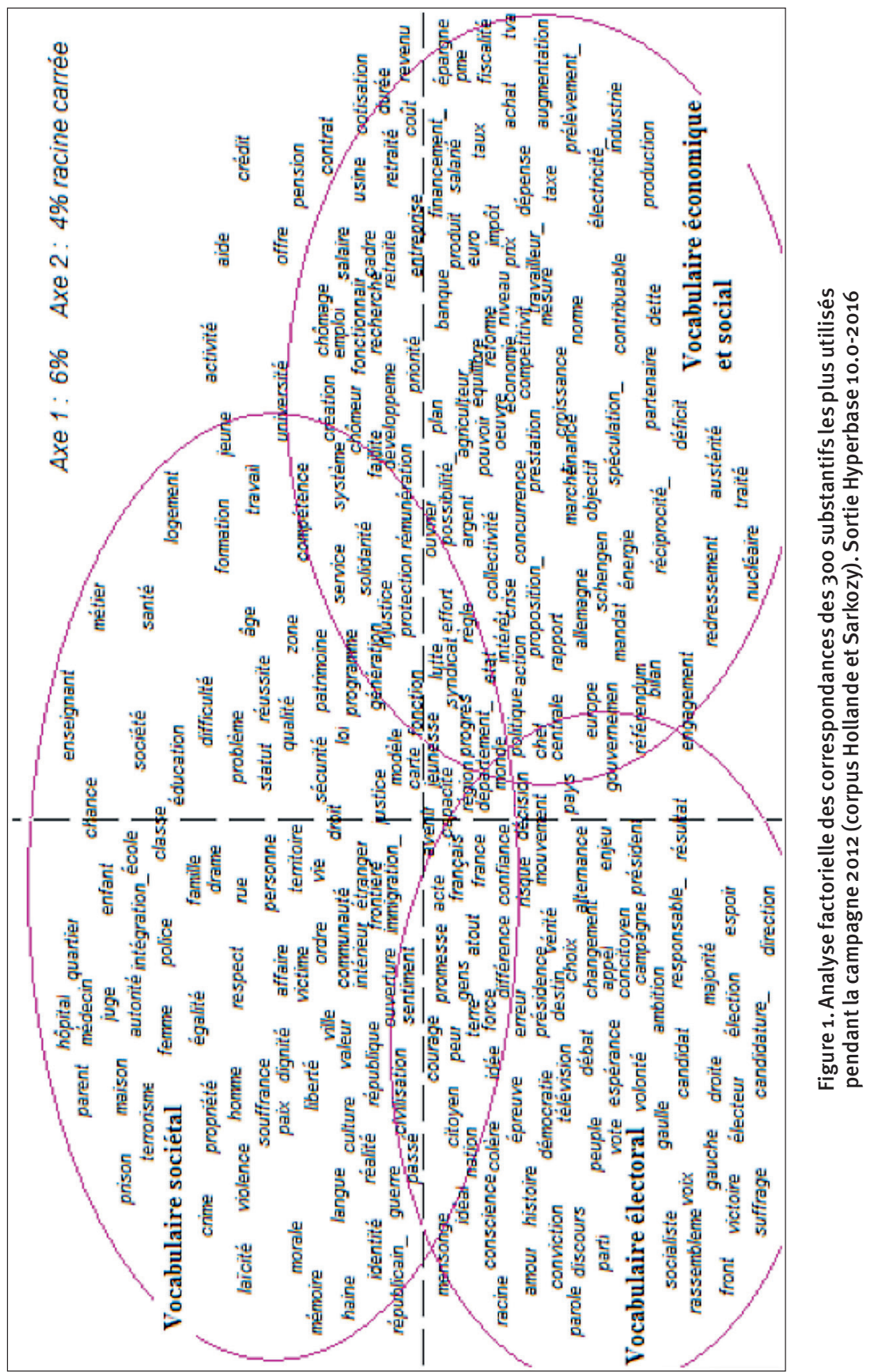




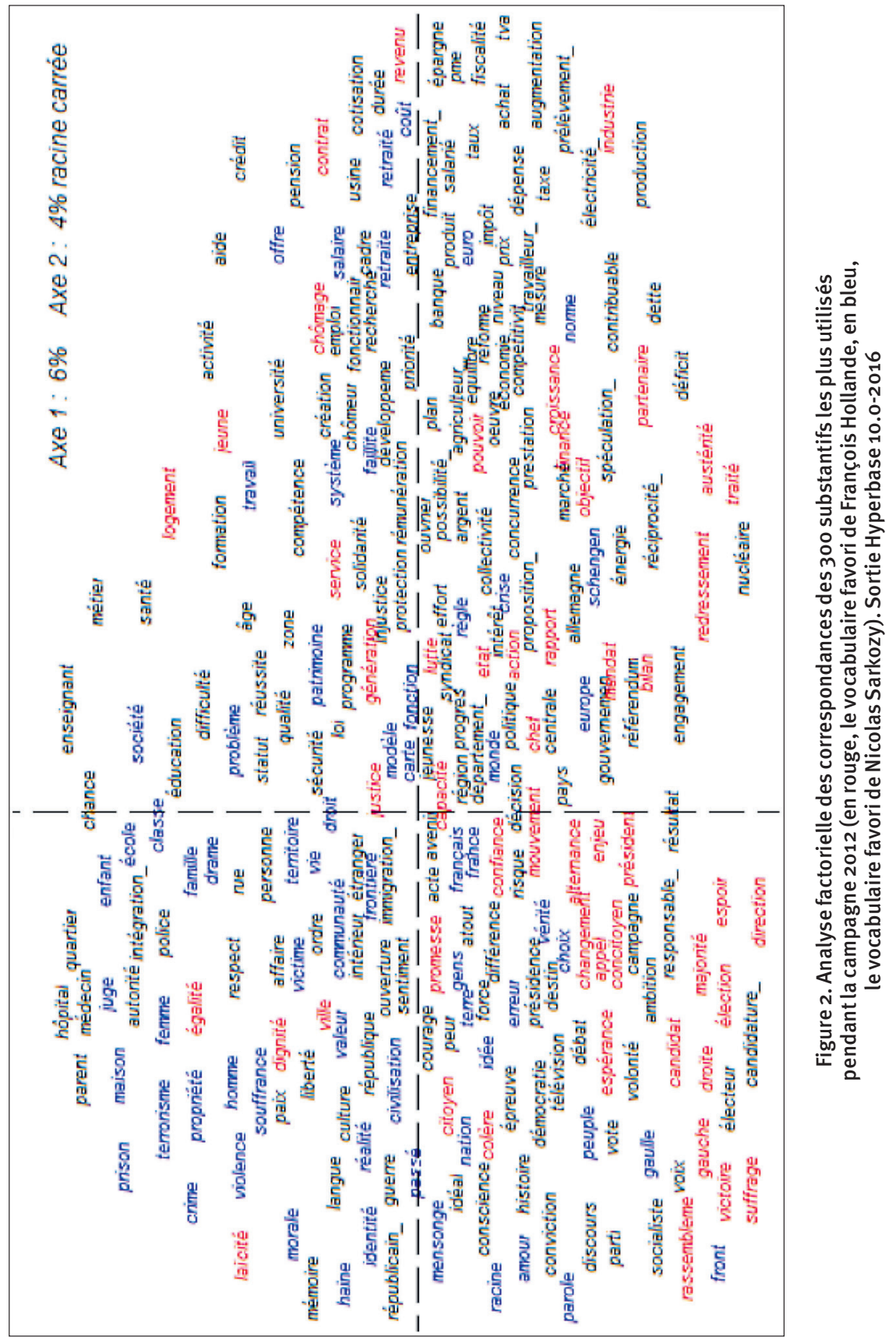


tues ou générales sur la société française avec par exemple les mots sociétaux de la santé (médecin, hôpital), de l'éducation (école, enseignement, enfants ou famille) ou encore, de manière plus élargie, avec les mots du vivre ensemble républicain (langue, culture, laïcité, mémoire, valeur, etc.)

Enfin une thématique électorale autour des enjeux politiques de l'élection se dessine nettement en bas à gauche du graphique avec par exemple les mots candidature, suffrage, électeur, voix, campagne.

Remarquons-le, la politique étrangère semble négligée, sans doute parce qu'elle est moins porteuse électoralement. Et rares sont les mots qui s'y rapportent malgré quelques mots-exceptions comme Europe, Allemagne ou monde; plus significatif encore de cette faiblesse de l'international dans le discours de campagne, si le monde intervient, c'est par l'intermédiaire de la frontière ou de l'immigration; les élections présidentielles, toutefois celle de 2012, semblent plutôt favoriser le repli sur soi et l'inquiétude identitaire que l'ouverture au monde.

L'affrontement. Si les deux candidats chevauchent ensemble ces trois thématiques dans une intertextualité et un dialogisme inévitables (chacun répondant nécessairement à l'autre), l'importance qu'ils apportent aux unes et aux autres diffère.

Sur le même graphique que précédemment, il est facile de projeter les spécificités (i. e. le vocabulaire caractéristique des deux candidats) 5 : en bleu, le vocabulaire privilégié de Nicolas Sarkozy et en rouge, celui de François Hollande; en noir, le vocabulaire équitablement partagé entre les deux.

Grosso modo, et à quelques intéressantes exceptions près, François Hollande favorise le vocabulaire politique et électoral, d'une part, et le vocabulaire économique et social, d'autre part. Par cette stratégie, il renvoie Nicolas Sarkozy à son bilan (le mot est très caractéristique) de sortant d'un point de vue politique (alternance, rassemblement, majorité, gauche, droite) et à son bilan économique - c'est-à-dire de son point de vue à ses échecs : austérité, redressement nécessaire, faiblesse de la croissance, montée du chômage, crise du logement, etc. C'est dans ce vocabulaire économique, omniprésent chez François Hollande, que l'on trouvera par exemple répété finance comme l'opinion publique le retiendra sur le moment comme après la campagne.

Nicolas Sarkozy de son côté essaye d'éviter ces sujets - il ne veut apparaitre ni comme le président sortant ni comme le responsable de la crise pour insister sur des questions sociétales voire civilisationnelles, comme la menace que représente le terrorisme, pour notre civilisation par exemple, comme la question de la restauration de la morale notamment à l'école, déjà

5. Les «spécificités» sont l’indice statistique majeur de la logométrie (précédemment de la lexicométrie) depuis les développements saint-clousiens, par exemple ici dans le premier numéro de Mots (Lafon, 1980). 
point d'orgue de sa campagne 2007 (Mayaffre, 2012b); comme ses réflexions patriotiques sur la France ou les Français, sur le passé glorieux de la nation, etc.

Sans que la statistique ne puisse rien prévoir en la matière, émettons l'hypothèse que le sortant Hollande, s'il devait se présenter en 2017, prendrait les habits discursifs de Sarkozy 2012 (esquive du bilan économique et du positionnement politique; mise en valeur des thématiques sociétales), là où le challenger de droite (peut-être à nouveau Sarkozy?) labourera la question du bilan économique et politique du président en exercice.

\section{Le Rubicon de l’Élysée?}

Que ce soit les analystes du discours qui insistent sur l'importance des genres discursifs ou le corps électoral qui soupçonne facilement les hommes politiques de revirement, tout le monde semble convenir que les politiciens changent leur discours aussitôt élus.

L'étude que l'on se propose de faire confronte douze sous-corpus : les discours de campagne de Hollande 2012 (1); les discours du président Hollande en 2012 (2), 2013 (3), 2014 (4) et 2015 (5) ; les discours de campagne de Sarkozy en 2007 (6) et en 2012 (7); et les discours du président Sarkozy durant les cinq années de mandat $(8,9,10,11,12)$.

Le calcul de la distance intertextuelle mesure la proximité/éloignement des textes deux à deux et la représentation arborée rend compte de la distance de tous à chacun ${ }^{6}$. Deux conclusions fortes doivent être tirées de la figure.

Le candidat et le président Hollande parlent d'une manière apparentée et, par là, contrastent avec le candidat et le président Sarkozy. La structuration de l'arbre atteste que la variable la plus importante d'une prise de parole n'est ni la chronologie (2007 versus 2012 par exemple), ni le genre du discours (discours de campagne versus discours de président), mais l'identité ou individuation politique du locuteur. Le locuteur Hollande, en haut de l'arbre, reste le locuteur Hollande quelles que soient les circonstances; personne - ni le citoyen ni Hyperbase - ne peut s'y tromper. Et l'orateur Sarkozy, en bas de l'arbre, reste l'orateur Sarkozy.

Pour autant, et cette deuxième conclusion vient nuancer la première, les discours de campagne s'originalisent par rapport aux discours présidentiels. C'est le cas pour François Hollande dont la branche «Hollande-Candidat-2012 » se distingue du bouquet de ses discours présidentiels. C'est le cas de manière plus spectaculaire avec Nicolas Sarkozy puisque une branche ad hoc de l'arbre rassemble, en dépit de la chronologie, les discours de campagne 2007 et 2012

6. La méthode et le calcul, largement éprouvés depuis, ont été exposés dans le détail dans un numéro spécial de la revue Corpus (2003). 


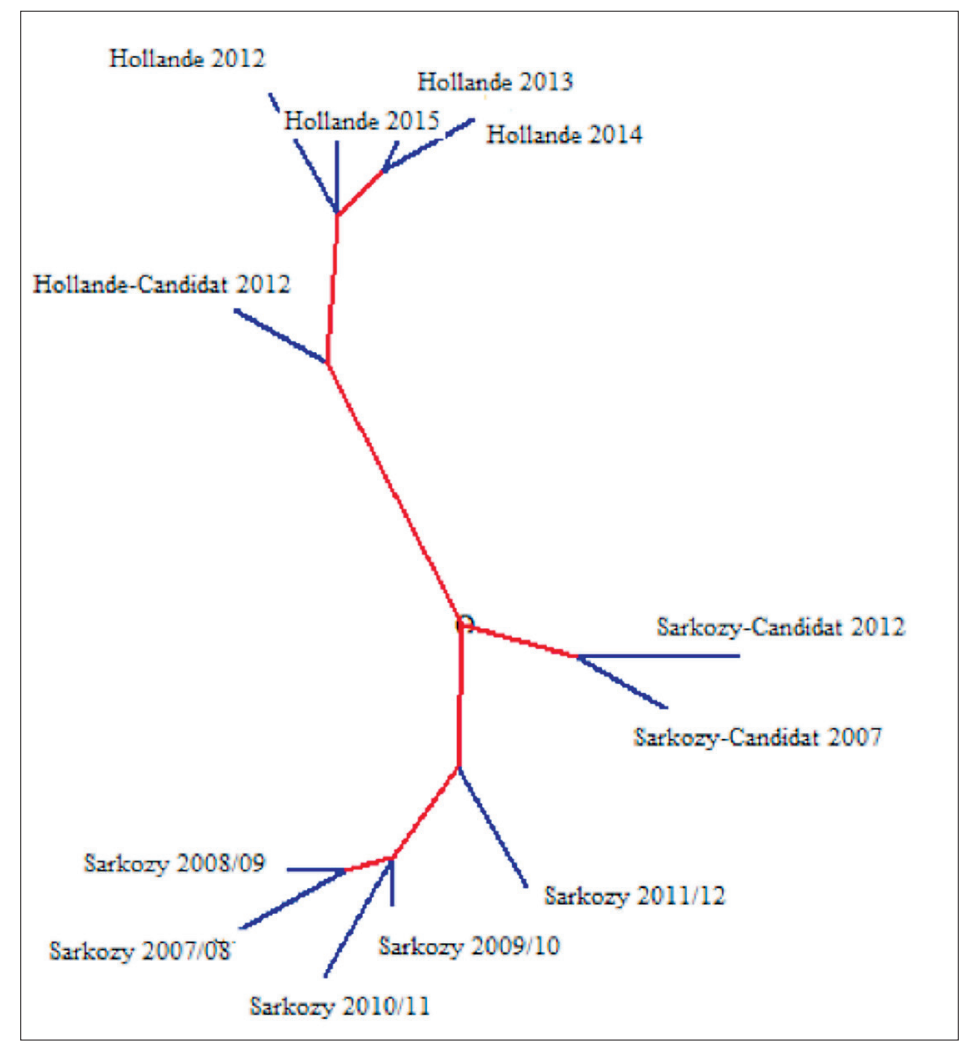

Figure 3. Représentation arborée de la distance lexicale entre les textes (corpus candidats/présidents). Sortie Hyperbase Web Edition

pour les distinguer de ses discours de président. Succinctement, sans que l'on puisse développer ici, les discours de campagne (Hollande 2012, Sarkozy 2007 et Sarkozy 2012) versus les discours des présidents se caractérisent, en quelques mots, comme suit :

\begin{tabular}{l|l}
\hline Spécificités +++ des présidentielles & Spécificités +++ des présidents \\
\hline $\begin{array}{l}\text { je, veux, France, ne... pas, enfants, nation, } \\
\text { candidat, peuple, culture, valeur, etc. }\end{array}$ & $\begin{array}{l}\text { gouvernement, entreprises, résultats, } \\
\text { croissance, Syrie, réforme, etc. }\end{array}$ \\
\hline
\end{tabular}

Dans la grande tradition gaullienne, le discours des présidentielles met en scène la rencontre entre un homme (je, veux) et le pays (France, nation, peuple) sans hésiter à faire des détours par les valeurs et principes (culture, valeur); c'est aussi un discours polémique avec les traces de la négation (ne... pas). Le discours présidentiel quant à lui est devenu, on le sait (voir infra), un discours 
avant tout économique (entreprise, croissance, résultats) et parfois de politique internationale (Syrie).

Quoi qu'il en soit, nous suggérons que le malaise démocratique dont la traduction historique la plus forte est l'augmentation quasi continue de l'abstention réside non pas fondamentalement dans la trahison discursive des politiques (le discours de Hollande reste un discours de Hollande par-delà l'élection) mais dans le divorce ressenti entre les discours-promissifs de campagne qui peuvent rester à la surface des mots et les discours-performatifs présidentiels dont l'électorat touche les limites en termes de réalisation concrète (notamment sur le terrain économique). C'est bien l'impuissance des politiques (leur capacité à agir) qui semble sanctionnée : la performativité du langage, satisfaisante lors d'une campagne électorale, devient insatisfaisante une fois élu; aux yeux des Français, promettre ou vouloir que le chômage baisse est un acte de langage suffisant pour un candidat mais devient insuffisant pour un président en exercice.

\section{Hollande et le discours présidentiel (1958-2016)}

Le dernier regard portera sur Hollande président, sur son discours à l'Élysée et sur ses caractéristiques historiques par rapport à celles de ses prédécesseurs. De fait, les observateurs peinent à accorder au président une personnalité discursive nette et l'ordinateur doit nous permettre de distinguer les mots-thèmes qui le caractérisent désormais en quatre ans de présidence?.

L'arbre de distance montre avant tout la pesanteur de la conjoncture historique sur le discours hollandais. Malgré un tempérament différent de celui de Nicolas Sarkozy et une culture politique autre, François Hollande n'arrive pas à se distinguer du discours de son immédiat prédécesseur. Calculé sur le lexique utilisé, la distance proche entre les deux derniers présidents s'explique avant tout par le vocabulaire économique de crise que l'un et l'autre surutilisent (banque, crise, dette, etc.). Dans les deux cas, nous sommes dans des discours de président-Premier ministre, voire ministre de l'Économie ou des Finances. Dans les deux cas, nous sommes même devant un discours du PDG de «l'entreprise France»; dans le deux cas ladite entreprise semble proche du dépôt de bilan et la question de l'avenir des ressources humaines semble posée (retraite, chômage, allocation, jeune, etc.).

Enfin, au-delà des ressemblances conjoncturelles avec Sarkozy, nous synthétisons pour conclure quelques caractéristiques du discours hollandais, que cela soit en matière de formes graphiques (mots), de lemmes, de codes gram-

7. Il convient de noter que l'étude du discours de Hollande est ici synchronique. Les quatre années ont été fondues dans un corpus unique ignorant les évolutions du propos hollandais au fil des événements qui ont saisi la France; comme nous ignorons les événements qui ont scandé les mandats successifs de De Gaulle, Pompidou, Giscard, Mitterrand, Chirac et Sarkozy. 


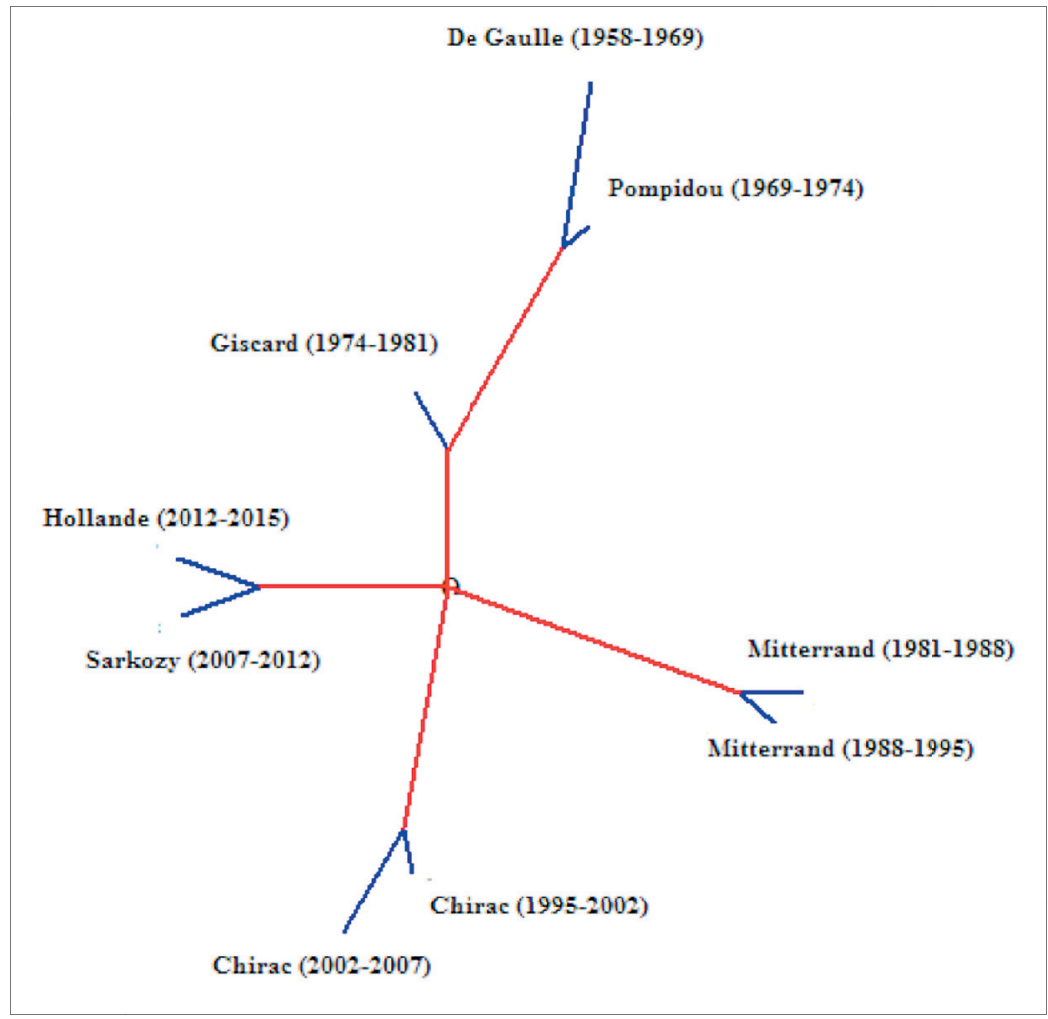

Figure 4. Représentation arborée de la distance lexicale entre les textes (corpus élyséen 1958-2015). Sortie Hyperbase Web Edition

\begin{tabular}{|c|c|c|}
\hline Forme graphiques & Lemmes & Codes \\
\hline Entreprises $(+16,6)$ & Est-ce que $(+21,1)$ & Ponctuation forte $(+18,5)$ \\
\hline Syrie $(+14,4)$ & Emploi $(+17,4)$ & $\begin{array}{l}\text { Nom commun Mascul Sing } \\
(+14,5)\end{array}$ \\
\hline Compétitivité $(+14,2)$ & Entreprise (subst.) $(+16,4)$ & $\begin{array}{l}\text { Déterminant Article Pluriel } \\
(+14,5)\end{array}$ \\
\hline bancaire $(+13,9)$ & Euros $(+14,5)$ & Verbe principal Infinitif $(+14,3)$ \\
\hline Mali $(+12,4)$ & Devoir (verbe) $(+12,2)$ & Pronom démonstratif $(+13,2)$ \\
\hline \multicolumn{2}{|c|}{ Segments répétés (longueur 9 et 8) } & Paires co-occurrentielles \\
\hline \multicolumn{2}{|c|}{... le contrat de génération qui va donner une... $(+14,3)$} & Avenir_Emploi $(+13,7)$ \\
\hline \multicolumn{2}{|c|}{... l’accord sur la sécurisation de l'emploi... $(+13,4)$} & Contrat_Génération $(+12,8)$ \\
\hline \multicolumn{2}{|c|}{... le barème de l'impôt sur le revenu...(+8) } & Emploi_Jeune $(+9,1)$ \\
\hline \multicolumn{2}{|c|}{... c'est le rôle du président de la république... $(+7,5)$} & Entreprise_Salarié $(+8,9)$ \\
\hline \multicolumn{2}{|c|}{...il n’y a pas de temps à perdre... $(+7,5)$} & Guerre_Syrie $(+7,6)$ \\
\hline
\end{tabular}

Figure 5. De quelques spécificités de François Hollande par rapport au corpus présidentiel (1958-2015). Sortie Hyperbase 10.0-2016 
maticaux, de segments répétés ou de paires cooccurrentielles.

Ce rapide panorama aura montré à grands traits la structuration thématique des discours de François Hollande en tant que candidat et en tant que président.

Nécessairement marqué par les conditions d'énonciation (campagne électorale versus exercice du pouvoir), le discours varie, mais il varie dans des proportions que les analyses des genres ont tendance à exagérer.

Que cela soit durant la campagne ou durant sa présidence, le discours de François Hollande est largement dominé par les considérations économiques. Certes, la politique internationale vient à l'agenda du président (particulièrement en 2015 après les attentats des 11 janvier et 13 novembre), là où elle est massivement ignorée du candidat. Certes encore, le candidat sait jouer du vocabulaire clivant de la politique là où le président évite le dissensus et l'affrontement politicien pour s'adresser à tous. Mais le paradigme économique, omniprésent, fait le pont entre la campagne et la présidence pour donner une unité au tout.

Ce tropisme économique doit être analysé historiquement, et ici la comparaison avec l'épopée longue du discours sous la Cinquième République apporte un témoignage utile (Mayaffre, 2012a; voir l'observatoire du discours présidentiel en ligne : 〈http://mesure-du-discours.unice.fr/〉, consulté le 20 juin 2016). Progressivement introduit par Valéry Giscard d'Estaing lors de la crise pétrolière, puis par François Mitterrand avec la question du chômage qui traverse ses septennats, le discours économique, minoritaire sous de Gaulle ou Pompidou, phagocyte aujourd'hui la grande majorité du discours. La crise du politique semble synonyme, dans le périmètre du discours, d'embellie pour l'économique. Le temps des gestionnaires semble avoir sonné à l'Élysée, avec des présidents devenus grand trésorier ou manager de la très grande entreprise France. L'orateur politique que la rhétorique a appris à décrire de Périclès à Robespierre, de Jaurès à de Gaulle, laisse ainsi la place à l'orateur œeconomicus qu'il reste encore à définir.

\section{Références}

Barbet Denis et Mayaffre Damon éd., 2009, Mots. Les Langages du politique, n89, «2007. Débats pour l’Élysée».

Ben Hamed Mahé et Mayaffre Damon éd., 2015, Mots. Les Langages du politique, n 108 , "Thèmes et thématiques dans le discours politique ».

BENZÉCRI Jean-Paul, 1973, L'analyse des données, t. II : L'analyse des correspondances, Paris, Dunod.

BouRdieu Pierre, 1982, Ce que parlerveut dire. L'économie des échanges linguistiques, Paris, Fayard.

Carcassonne Guy, 2011, La Constitution, Paris, Le Seuil. 
CORPUS, 2003, $\mathrm{n}^{\circ} 2$, "La distance intertextuelle».

GUARESI Magali, 2015, "Les thèmes dans le discours électoral de candidature à la députation sous la Cinquième République. Perspective de genre (1958-2007)», Mots. Les langages du politique, $\mathrm{n}^{0} 108$, «Thèmes et thématiques dans le discours politique », p. 15-37.

LABBÉ Dominique, Monıère Denis, 2013, La campagne présidentielle de 2012. Votez pour moi, Paris, L'Harmattan.

LAFON Pierre, 1980, "Sur la variabilité de la fréquence des formes dans un corpus», Mots. Mots, ordinateurs, textes, sociétés : travaux de lexicométrie et de lexicologie politique, $\mathrm{n}^{0} 1$, «Saussure, Zipf, Lagado, des méthodes, des calculs, des doutes et le vocabulaire de quelques textes politiques »,p.127-165.

McСомвS Maxwell, SHAw Donald, 1972, "The agenda-setting function of mass media », Public Opinion Quarterly, vol. 36, n² 2, p. 176-187.

Mayaffre Damon, 2012a, Le discours présidentiel sous la Ve République. Chirac, Mitterrand, Giscard, Pompidou, de Gaulle, Paris, Presses de Sciences Po.

- 2012b, Mesure et démesure du discours. Nicolas Sarkozy (2007-2012), Paris, Presses de Sciences Po.

Mots. Les langages du politique, 2009, n89: «2007. Débats pour l’Élysée». NÉE Émilie, 2012, L’Insécurité en campagne électorale, Paris, Honoré Champion.

VIPREY Jean-Marie, 2006, "Structure non-séquentielle des textes ", Langages, nº 163 , p. $71-85$. 